\title{
Metabolic engineering of yeast to produce fatty acid-derived biofuels: bottlenecks and solutions
}

\author{
Jiayuan Sheng and Xueyang Feng * \\ Biomolecular Engineering Lab, Department of Biological Systems Engineering, Virginia Polytechnic Institute and State \\ University, Blacksburg, VA, USA
}

Fatty acid-derived biofuels can be a better solution than bioethanol to replace petroleum fuel, since they have similar energy content and combustion properties as current transportation fuels. The environmentally friendly microbial fermentation process has been used to synthesize advanced biofuels from renewable feedstock. Due to their robustness as well as the high tolerance to fermentation inhibitors and phage contamination, yeast strains such as Saccharomyces cerevisiae and Yarrowia lipolytica have attracted tremendous attention in recent studies regarding the production of

OPEN ACCESS

Edited by:

Aindrila Mukhopadhyay,

Lawrence Berkeley National

Laboratory, USA

Reviewed by:

Marina G. Kalyuzhanaya, San Diego State University, USA Leo D'Espaux

Lawrence Berkeley National Laboratory, USA

*Correspondence: Xueyang Feng, Virginia Polytechnic Institute and State University, 1230 Washington St. SW, Blacksburg, 24061 VA, USA xueyang@vt.edu

Specialty section:

This article was submitted to Microbial Physiology and Metabolism, a section of the journal Frontiers in Microbiology

Received: 19 January 2015 Accepted: 20 May 2015

Published: 08 June 2015

Citation:

Sheng $J$ and Feng $X$ (2015) Metabolic engineering of yeast to produce fatty acid-derived biofuels: bottlenecks and solutions. Front. Microbiol. 6:554. doi: 10.3389/fmicb.2015.00554 fatty acid-derived biofuels, including fatty acids, fatty acid ethyl esters, fatty alcohols, and fatty alkanes. However, the native yeast strains cannot produce fatty acids and fatty acid-derived biofuels in large quantities. To this end, we have summarized recent publications in this review on metabolic engineering of yeast strains to improve the production of fatty acid-derived biofuels, identified the bottlenecks that limit the productivity of biofuels, and categorized the appropriate approaches to overcome these obstacles.

\section{Keywords: fatty acid biosynthesis, S. cerevisiae, Y. lipolytica, acetyl-CoA, metabolic regulation}

\section{Introduction}

The industry of fuel production continues to grow at an increasing rate each year. Currently, around one third of fuels are derived from petroleum feedstock worldwide while the rest are from coal, natural gas, nuclear energy, hydro-electricity and renewables (BP, 2014). Around $80 \%$ of the liquid transportation fuels are derived from petroleum (Floudas et al., 2012). The rapidly depleting petroleum reservoirs and growing environmental concerns on using petroleum feedstock make it increasingly important to shift to a renewable-based industry (Zhang et al., 2011a). Among various forms of renewable energy, microbial production of biofuels, despite drawbacks such as low benefit-cost ratio and scale-up issues, stands as a promising replacement of petroleum fuel since they are environmentally and economically friendly. First generation biofuels, such as bioethanol fermented from corn and biodiesel esterified from edible vegetable oils or animal fats, cover over $90 \%$ of the current biofuel market (Antoni et al., 2007). This kind of replacement of petroleum fuel would require occupying huge amounts of farmland for biofuel production, which would challenge the world food supply and cause a series of economic problems (Peralta-Yahya et al., 2012). Therefore, second generation biofuels, such as the advanced biofuels, can be a better solution, since they can be produced from low-cost agricultural byproducts, including wheat straw, forest waste, and energy crops (e.g., switchgrass) (Zhang et al., 2011a). Proposed advanced fuels include butanol (Connor and Liao, 2009; Yan and Liao, 2009), isopentanol (Zhang et al., 2011a), terpenes 
(Peralta-Yahya et al., 2011; Zhang et al., 2014), fatty acid ethyl esters (FAEEs) (Fukuda et al., 2001; Steen et al., 2010), and alkanes (Schirmer et al., 2010; Peralta-Yahya et al., 2012), which have very similar energy content, storage and transportation properties, and combustion properties as those in current transportation fuels, allowing these advanced biofuels to be directly used in existing gasoline, diesel, and jet engines (Hamelinck and Faaij, 2006; Peralta-Yahya et al., 2012).

Among the advanced biofuels, fatty acid-derived compounds, including free fatty acids (FFAs), fatty alcohols, FAEEs, or fatty acid methyl esters (FAMEs), and fatty alkane/alkenes, are of particular interest, since they fulfill a role as platform molecules of a cluster of important fuels. FFAs serve as precursors to soaps, surfactants, and lubricants (Tee et al., 2014) and can be converted to fatty alcohols, FAEEs and FAMEs. Fatty alcohols are widely used in detergents, skin care products, cosmetics, and medicines, and are also considered to be potential biofuels (Liu et al., 2014). FAEE and FAME are excellent diesel fuel replacements due to their low water solubility and high energy density, and are suitable for microbial production because of their low toxicity to host cells (Zhang et al., 2012). Alkanes, observed throughout nature, are the major constituents of gasoline, diesel, and jet fuel (Kissin, 1987). The natural fatty acid market was valued at $\$ 7.2$ billion in 2011 and the total market value is expected to reach $\$ 13$ billion in 2017 after increasing at a 5-year compound annual growth rate (CAGR) of $13.6 \%$ (BCC Research LLC, 2013). The applications of fatty acid derivatives had a value of nearly $\$ 3.3$ billion in 2012, and are expected to have a value of $\$ 6.5$ billion in 2017 (BCC Research LLC, 2013).

The microbial production of fatty acid-derived biofuels have recently been achieved in many industrial applications (Trotter, 2001; Strijbis and Distel, 2010; Lennen and Pfleger, 2013; Jones et al., 2014; Lee et al., 2014; Beld et al., 2015; Jia et al., 2015; Pasztor et al., 2015; Pfleger et al., 2015). There have been numerous literatures focusing on the overproduction of fatty acid-derived biofuels in the past 5 years, most of which have used Escherichia coli as the host (Steen et al., 2010; Zhang et al., 2011b; Youngquist et al., 2012; Xu et al., 2013; Jones et al., 2014; Chu et al., 2015; Clomburg et al., 2015; Liu et al., 2015a; Tai et al., 2015). Although the number of literatures published on fatty acid production using yeast such as S. cerevisiae and Yarrowia lipolytica is far more limited than that of $E$. coli, yeast has its own advantages which make it an appealing host. First, the synthetic route of fatty acids is more direct and shorter than that of E. coli, which allows more efficient conversion of carbon substrate to fatty acids and fatty acid-derived biofuels (Zhang et al., 2011a; Runguphan and Keasling, 2014). Also, yeast is a robust industrial organism that can grow under low $\mathrm{pH}$ levels and various harsh fermentation conditions and is free of phage contamination (Hong and Nielsen, 2012; Runguphan and Keasling, 2014). Many of the yeast strains have been fully sequenced and well characterized for their metabolic pathways with the availability of a cluster of genetic tools (Hong and Nielsen, 2012). Recently, there has been an increasing interest in developing yeast as a cell factory for fatty acid-derived biofuel production. However, engineering yeast to produce fatty acid-derived biofuels also faces many challenges. In fact, the highest titer of fatty acids, fatty alcohol and FAEEs could only reach $2.2,1.1$, and $0.52 \mathrm{~g} / \mathrm{L}$, respectively, in yeast (Table 1 ),
TABLE 1 | Comparison of the productivity of fatty acid-derived biofuels between $E$. coli and S. cerevisiae.

\begin{tabular}{|c|c|c|c|c|}
\hline & \multicolumn{2}{|r|}{ E. coli } & \multicolumn{2}{|c|}{ S. cerevisiae } \\
\hline & Titer (g/L) & References & Titer (g/L) & References \\
\hline Fatty acids & 8.6 & Xu et al., 2013 & 2.2 & $\begin{array}{l}\text { Cardenas and Da } \\
\text { Silva, } 2014\end{array}$ \\
\hline $\begin{array}{l}\text { Fatty } \\
\text { alcohols }\end{array}$ & 1.95 & Cao et al., 2015 & 1.1 & Feng et al., 2014 \\
\hline FAEEs & 11 & $\begin{array}{l}\text { Elbahloul and } \\
\text { Steinbuchel, } 2010\end{array}$ & 0.52 & Yu et al., 2012 \\
\hline Fatty alkanes & 0.58 & $\begin{array}{l}\text { Choi and Lee, } \\
2013\end{array}$ & $0.0001^{*}$ & Buijs et al., 2014 \\
\hline
\end{tabular}

*The data was calculated based on the assumption of biomass was $5 \mathrm{~g} / \mathrm{L}$. The original data was $22.0 \pm 1.4 \mu \mathrm{g} / \mathrm{g} \mathrm{DCW}$.

which is even lower than that of $E$. coli $(8.6,1.7$, and $11 \mathrm{~g} / \mathrm{L}$, respectively). Long-chain alkane production in yeast has not been successfully achieved until a very recent research breakthrough in metabolic engineering of $S$. cerevisiae, which, however, can only produce $13.5 \mu \mathrm{g} / \mathrm{L}$ heptadecane (Buijs et al., 2014).

In this review, we summarized the research achievements that have been accomplished in the past 5 years on engineering yeast (mostly S. cerevisiae and Y. lipolytica) to produce fatty acid-derived biofuels (Table 2). We focused on discussing the limitations that have been discovered by many of the researchers in the area of metabolic engineering when tuning the fatty acid metabolism in yeast strains. We then summarized the synthetic and systems biology approaches to overcome these difficulties. We also identified several potential issues that have not yet been well addressed when modifying yeast metabolism for improved production of fatty acid-derived biofuels.

\section{Bottlenecks of Producing Fatty Acid-Derived Biofuels in Yeast and Their Solutions}

To produce fatty acid-derived biofuels, the fatty acid biosynthesis (FAB) pathway needs to be appropriately engineered. FAB begins with the conversion of acetyl-CoA into malonyl-CoA by acetyl-CoA carboxylase (ACC). Then these two precursors are condensed by fatty acid synthases (FASs) to fatty acids using the malonyl-CoA as the extender unit. Each elongation of two carbon unit in FAB costs $2 \mathrm{NADPH}$. In the cytosol of $S$. cerevisiae, FAB is catalyzed by type I FAS system, with all the functional domains organized into two subunits, encoded by FAS1 ( $\beta$-subunit) and FAS2 ( $\alpha$-subunit) (Hiltunen et al., 2009; Chan and Vogel, 2010). Since all functional domains, including the ACP, are organized in the FAS complex, the whole FAB process is performed within the fatty acid elongation chamber after malonyl-CoA is loaded. The released fatty acyl-CoAs can be converted to the desired products, such as FFAs, fatty alcohols, and FAEEs by the corresponding terminal enzymes. However, S. cerevisiae naturally produces fatty acids at very low levels due to a series of obstacles (Lian and Zhao, 2014). Here in this review, we have summarized over 100 publications on metabolic engineering of yeast fatty acid metabolism and listed the bottlenecks into five categories: (1) 
TABLE 2 | Metabolic engineering of yeast to improve the production of fatty acid-derived biofuels.

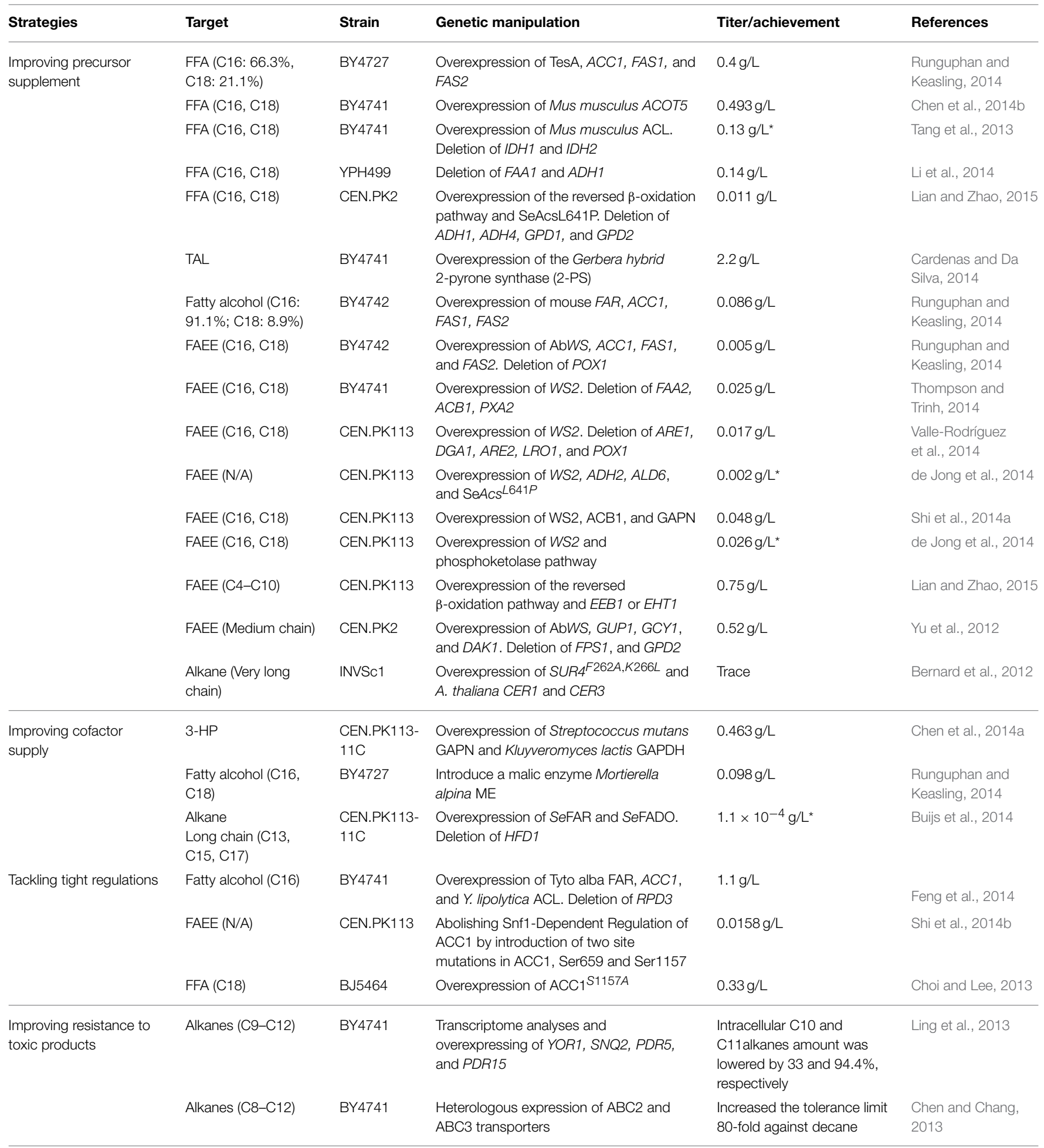

${ }^{*}$ Calculated based on the assumption that the final biomass was $5 \mathrm{~g} / \mathrm{L}$.

the limited supply of FAB precursor; (2) the limited supply of cofactors; (3) the tight regulations of fatty acid metabolism; (4) the product toxicity; and (5) the lack of metabolic engineering tools in non-model oleaginous yeast.

\section{The Limited Supply of FAB Precursors}

In $S$. cerevisiae, the synthesis of fatty acids can take place in at least two subcellular compartments: cytoplasm (type I FAS) and mitochondria (type II FAS). The cytosol type I FAS produces most 
of the fatty acids in the cell, which indicates that the predominant efforts on metabolic engineering of fatty acid synthesis should be focused on improving the supply of cytosolic precursors (i.e., acetyl-CoA, malony-CoA, and fatty acyl-CoA) for fatty acid synthesis.

Acetyl-CoA is a key precursor in the biosynthesis of sterols, amino acids, fatty acids, and polyketides (Tai and Stephanopoulos, 2013; Lian et al., 2014; Shi and Tu, 2015). In $S$. cerevisiae, acetyl-CoA metabolism takes place in at least four subcellular compartments: nucleus, mitochondria, cytosol and peroxisomes. Since fatty acids are mostly synthesized in the cytosol of $S$. cerevisiae, the pyruvate-acetaldehyde-acetate pathway (PDC pathway) is one of the pathways used to produce acetyl-CoA for fatty acid synthesis (Chen et al., 2012). The cytosolic acetyl-CoA is tightly controlled (Pfeiffer and Morley, 2014), probably limiting in high glucose conditions (Postma et al., 1989; Chen et al., 2013) and separate from the other pools (Crabtree, 1928; Lian and Zhao, 2014), which is contrasted with bacterial acetyl-CoA (Takamura and Nomura, 1988; Cronan and Waldrop, 2002). The activation of acetate to acetyl-CoA was also found to be a rate-limiting step because of the feedback inhibition on ACS and the requirement of ATP input (Lian and Zhao, 2014).

Since the supplement of cytosol acetyl-CoA is very limited, the central metabolism of yeast should be engineered to redirect the metabolic flux to cytosolic acetyl-CoA biosynthesis (Krivoruchko et al., 2015). It has been found that deleting major ADHs in the cytosol $(\triangle A D H 1-\triangle A D H 4)$, acetyl-CoA level was increased around 2-fold (Lian et al., 2014). In another study, 1.9-fold improvement in fatty acids production was achieved by knocking out $A D H 1$ in a fatty acid-producing host (Li et al., 2014). Beside engineering host metabolism, numerous heterologous pathways, including cytosolic pyruvate dehydrogenase (PDH) (Jing et al., 2011; Chen et al., 2013), pyruvate:formate lyase (PFL) (Kozak et al., 2014), pyruvate:ferrodoxin oxidoreductase (PFO), pyruvate: $\mathrm{NADP}^{+}$oxidoreductase (PNO) (Inui et al., 1987), ATPdependent citrate lyase (ACL), (Zaidi et al., 2012), acetylating aldehyde dehydrogenase (A-ALD), and phosphoketolase pathway (PK) (Sonderegger et al., 2004; de Jong et al., 2014), were introduced in $S$. cerevisiae to enhance the acetyl-CoA level in the cytosol, leading to the improvement of fatty acid production by 1.17 -fold, n-butanol production by about 3 -fold and FAEE production by 5.7 -fold. In addition, the inactivation of acetyl-CoA consuming pathways could further increase the FAB activity. Glyoxylate shunt, which contributes to the transport and consumption of cytosolic acetyl-CoA in yeast, becomes the most important target (Chen et al., 2012; Tang et al., 2013). However, the combination of the "pull and push" engineering strategy failed to further increase the acetyl-CoA level. The unexpected results might be attributed to the accumulation of acetate to a cytotoxicity level that is accompanied with the decreased production of acetyl-CoA derived products (Lian et al., 2014).

Another key precursor in FAB, malonyl-CoA, is catalyzed from acetyl-CoA via acetyl-CoA carboxylase (ACC), which is encoded by the ACC1 gene in S. cerevisiae. This is the first step in FAB and is well known as the rate limiting step (Li et al., 2014; Runguphan and Keasling, 2014; Zhou et al., 2014). Therefore, the pathways for malonyl-CoA synthesis need to be well engineered to improve the availability of malonyl-CoA in yeast. The most straightforward strategy is to overexpress the $A C C 1$ gene and $F A S$ genes. When overexpressing all three FAB genes, ACC1, FAS1, and FAS2 in S. cerevisiae, the production of FFAs achieved at a titer of approximately $400 \mathrm{mg} / \mathrm{L}$, fatty alcohols at approximately $100 \mathrm{mg} / \mathrm{L}$ and FAEEs at approximately $5 \mathrm{mg} / \mathrm{L}$ directly from simple sugars (Runguphan and Keasling, 2014). Researchers also found that by introducing a heterologous FAS from Brevibacterium ammoniagenes, FAEE titer was increased by 6.3 -fold compared to strains without expressing the heterologous FAS (Eriksen et al., 2015). In addition to the overexpression of ACC1, direct synthesis of malonyl-CoA from malonate by the malonyl-CoA synthetase (MCS) could also lead to improved malonyl-CoA levels. Basically, malonate was added exogenously and transported by a heterologous dicarboxylic acid plasma membrane transporter that was introduced to S. cerevisiae (Chen and Tan, 2013). The MCS from plants (Wang et al., 2014) or bacteria (Chen and Tan, 2013) has been cloned, characterized and functionally expressed in S. cerevisiae. Overexpression of a plant malonylCoA synthetase gene $(A A E 13)$ in S. cerevisiae resulted in 1.6fold and 2.4-fold increases in lipid and resveratrol accumulation simultaneously (Wang et al., 2014). Considering the important role of malonyl-CoA as the precursor for the synthesis of a wide variety of value-added compounds (e.g., polyketides), malonate supplementation and MCS overexpression may be a possible route to improve cellular level of malonyl-CoA in yeast (Chen and Tan, 2013). However, in order to achieve the economic production of fatty acid-derived biofuels, the high cost of malonate (approximately $\$ 8000 /$ ton) needs to be taken into consideration.

In addition to acetyl-CoA and malonyl-CoA, another important precursor for synthesizing fatty acid-derived biofuels is fatty acyl-CoA. To improve the fatty acyl-CoA level, the competing pathway, the $\beta$-oxidation cycle, was disrupted in $S$. cerevisiae by deleting POX1, a key enzyme in the $\beta$-oxidation pathway. Unfortunately, the disruption of $\beta$-oxidation cycle failed to improve the production of fatty alcohols and FAEEs (Runguphan and Keasling, 2014). However, by combining the strategy of disrupting the acyl-CoA transport to the $\beta$-oxidation pathway, avoiding TAG biosynthesis, and utilizing nitrogen limiting culture conditions to push carbon toward fatty acyl-CoA production, cytosolic acyl-CoA pools have been increased for FAEEs biosynthesis, leading to over $25 \mathrm{mg} / \mathrm{L}$ FAEEs produced, a $40 \%$ improvement over previous reports (Valle-Rodríguez et al., 2014).

\section{The Limited Supply of Cofactors}

As shown in Figure 1, NADPH provides the reducing power during the FAB process. Generally, the elongation of the fatty acid will cost $2 \mathrm{NADPH}$ to incorporate a $\mathrm{C} 2$ unit. In addition to fatty acid synthesis, NADPH is also involved in many other biological functions, such as lipid synthesis, cholesterol synthesis, and amino acid synthesis. In $S$. cerevisiae, the generation of $\mathrm{NADPH}$ occurs in cytosol, mitochondria and peroxisome. In the cytosol, the oxidative pentose phosphate pathway generates the majority 


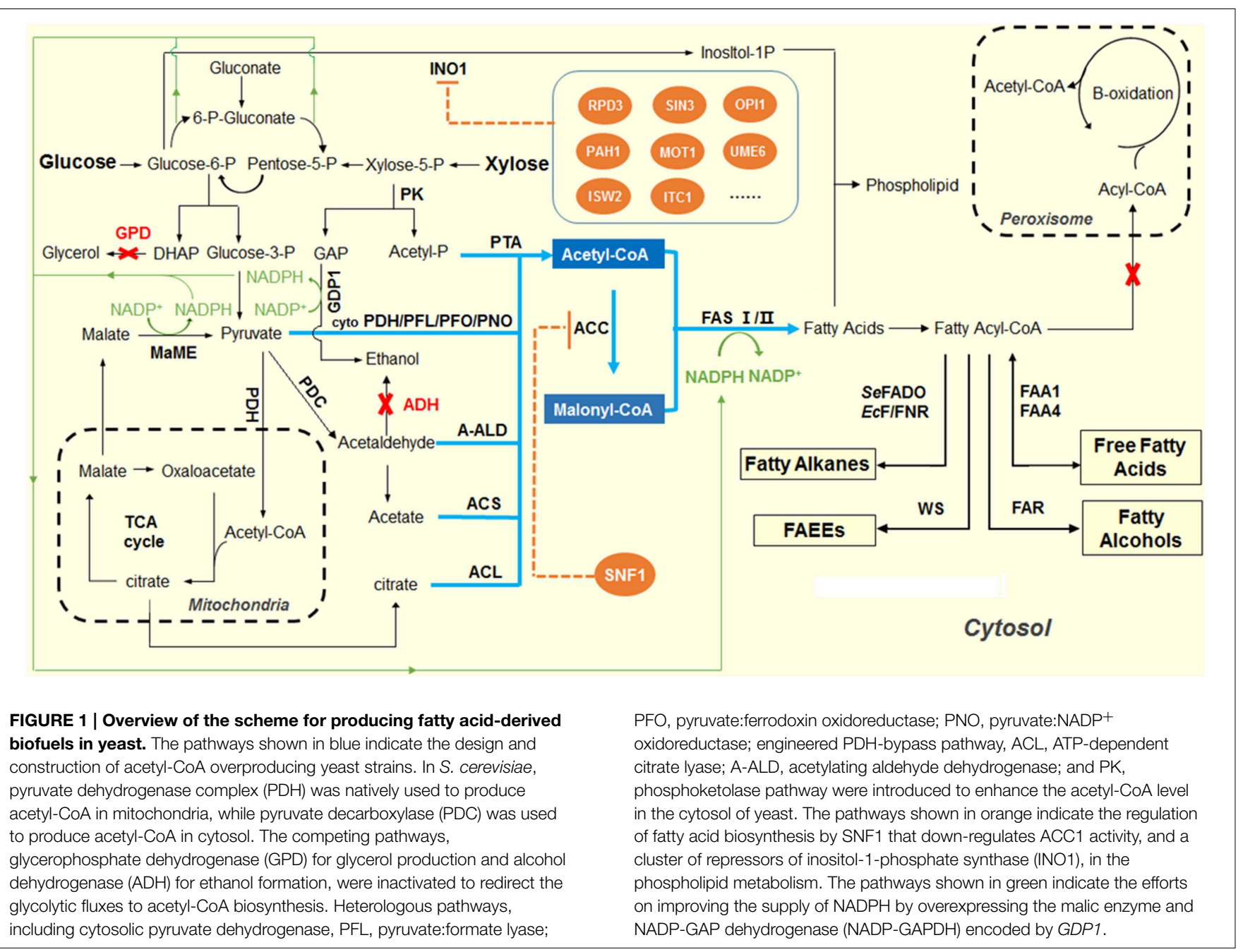

of NADPH (Bruinenberg et al., 1983; Minard and McAlisterHenn, 2005). In mitochondria, the isocitrate dehydrogenase (IPD2) in the TCA cycle could generate NADPH. Peroxisomal isocitrate dehydrogenase (IPD3) catalyzes oxidation of isocitrate to alpha-ketoglutarate with the formation of NADPH, which is required for growth on unsaturated fatty acids (Henke et al., 1998; van Roermund et al., 1998). As one of the major NADPHconsuming processes, the FAB is greatly constrained by the lack of NADPH supply in the cytosol.

In order to improve the NADPH supply in the cytosol, different strategies have been adopted. For example, a non-phosphorylating, $\mathrm{NADP}^{+}$-dependent glycerol dehyde3-phosphate dehydrogenase (GAPN), which catalyzes the irreversible oxidation of glyceraldehyde-3-phosphate and $\mathrm{NADP}^{+}$into 3-phosphoglycerate and NADPH in glycolysis, was heterologously expressed to increase the NADPH supply and improved the productivity of fatty acid-derived biofuels (Guo et al., 2011). The overexpression of GAPN together with wax ester synthase (WS2) resulted in a final titer for FAEEs of 148 $\mathrm{mg} / \mathrm{L}$, a $40 \%$ increase compared to the control strain (de Jong et al., 2014; Shi et al., 2014a). In another study, overexpression of
GAPN with enhanced malonyl-CoA supply led to a 70\% increase in 3-hydroxypropionic acid production (Chen et al., 2014a). Another reported approach is the heterologous expression of GDP1, a $\mathrm{NADP}^{+}$-dependent glyceraldehyde-3-phosphate dehydrogenase (NADP-GAPDH, EC1.2.1.13). The GDP1 from Kluyveromyces lactis was expressed in S. cerevisiae strain together with the NADPH-consuming fungal xylose pathway, and increased the xylose utilization with a higher ethanol productivity and yield compared to the control strain without expressing GDP1 (Verho et al., 2003).

In addition to GAPN and NADP dependent GAPDH, another common strategy to achieve NADPH overproduction is to overexpress an NADP-dependent malic enzyme. Malate is mainly synthesized as an intermediate in the citric acid cycle. Malic Enzyme converts malate and $\mathrm{NADP}^{+}$to pyruvate and $\mathrm{NADPH}$, releasing one molecule of carbon dioxide. Overexpressing the malic enzyme from the oleaginous fungus Mortierella alpina in the fatty alcohol-producing strain leads to a marginal increase (about 14\%) in the final fatty alcohol titer (Runguphan and Keasling, 2014). Due to the complicated generation and consumption pathways of NADPH in the metabolic network, 
new strategies need to be explored to increase the NADPH supply, which could further improve the productivity of fatty acid-derived biofuels.

\section{The Tight Regulations of Fatty Acid Metabolism}

Fatty acids are essential compounds in the cell that mainly serve a role as intermediates in lipid biosynthesis. Synthesis of fatty acids utilizes substantial amounts of metabolites, acetyl-CoA, ATP and NADPH, and may thus compete with other cellular processes dependent on these compounds. Due to the tight regulation of the FAS systems, S. cerevisiae does not naturally produce fatty acids to high levels to ensure proper fatty acid composition and homeostasis (Tehlivets et al., 2007). Fatty acid metabolism is regulated on both transcriptional and translational levels. As the key enzyme in FAB, the housekeeping enzyme FAS is expressed constitutively at a low level. It is found that some general yeast transcription factors (such as RAP1, ABF1, and REB1) (Schüller et al., 1994) and theinositol/choline-responsive transcription factor heterodimer (Ino2p and Ino4p) (Schüller et al., 1992) could activate its expression. Additionally, the FAS subunits are further regulated by proteolytic degradation of excess subunits. The intact FAS multimeric complex (alpha6beta6) is stable, but its individual subunits are rapidly degraded (Egner et al., 1993). Under such tight regulations on multilevels, the direct engineering of FAS was extremely difficult. Since only limited success was achieved in yeast to enhance fatty acid derived biofuel production via manipulating the structural genes, developing novel methods that can increase the flux toward producing fatty acid-derived biofuels becomes especially important. Two well-known families of transcriptional factors, SNF1 protein and the regulators of INO1, were focused in some of the recent reports since both families of the regulators could hamper the fatty acids synthesis via downregulation of either ACC1 or phospholipid synthesis (Feng et al., 2014).

Straightforward overexpression of the ACC1 gene can only improve the production of malonyl-CoA-derived molecules to a very limited extent. For example, less than 2 -fold improvements were achieved in producing polyketide 6-MSA, FAEEs and 3-hydroxypropionic acid, by exclusively overexpressing ACC1 (Wattanachaisaereekul et al., 2008; Shi et al., 2014b). Such results indicated the control of ACC1 enzyme activities is more complicated than gene overexpression, making it necessary to engineer the regulatory machinery. The ACC1 activity in $S$. cerevisiae is post-translationally regulated and repressed by various regulators. One of the most well-known regulators of ACC1 is SNF1, which not only represses the ACC1 activity, but also induces genes involved in gluconeogenesis, glyoxylate cycle, oxidation of fatty acids, as well as genes involved in the general stress response, pseudohyphal growth, aging, and ion homeostasis (Hong and Nielsen, 2012). The down-regulation of ACC1 activities by SNF1 could be eliminated by changing the phosphorylation recognition motif (Hyd-X-Arg-XX-SerXXX-Hyd) of SNF1. By mutating the putative phosphorylation sites from serine to alanine $\left(\mathrm{ACC1}^{\mathrm{S659A,S1157A}}\right.$ ), the ACC1 activity was increased by more than 3-fold and the production of malonyl-CoA derived metabolites, FAEEs and 3-HP, was increased by about 3.3- and 3.4-fold, respectively (Shi et al., 2014b).

The regulators of INO1, on the other hand, have been well known to transcriptionally control phospholipid synthesis by binding to cis-regulatory element $\mathrm{UAS}_{\mathrm{INO}}$ in the promoter region of the INO1 gene, which encodes inositol-1-phosphatesynthase as the first step for synthesis of inositol phosphates and inositol-containing phospholipids (Ambroziak and Henry, 1994; Feng et al., 2014). Since phospholipid synthesis requires fatty acids as precursors, it was expected that the deletion of negative regulators of phospholipid metabolism would enhance phospholipid production, and hence increase fatty acid synthesis. The family of regulators of INO1 includes a dozen transcription factors that interact with each other to control the expression level of the INO1 gene (Feng et al., 2014). In one of the recent studies, PAH1, RPD3, SIN3, OPI1, UME6, ITC1, ISW2, and MOT1 were chosen as the target regulators to knockout, since all of these transcription factors can decrease INO1 expression in phospholipid production. For $\triangle P A H 1, \triangle R P D 3$, $\triangle M O T 1$, and $\triangle O P I 1$, the production of the target chemical, 1-hexadecanol, was enhanced by $60-170 \%$, compared to the strain without knocking out the regulators. The highest titer was achieved at $122 \mathrm{mg} / \mathrm{L}$ in the $\triangle R P D 3$ strain (Feng et al., 2014).

\section{The Product Toxicity}

Hydrocarbons (e.g., alkanes) have been recently considered important next-generation biofuels. Several studies have demonstrated microbial production of alkane biofuels in both E. coli and yeast (Bernard et al., 2012; Choi and Lee, 2013; Buijs et al., 2014). Despite the promising potential of microbial alkane production, the yields and titers are key considerations for industrial-scale production. The toxicity of alkanes to microbial hosts can be a possible bottleneck for high productivity of alkane biofuels. Many studies have focused on finding the mechanism of the toxicity of fatty alkanes. It has been found that alkane products interact preferentially with cytoplasmic membrane, therefore disorganizing its structural integrity (Gill and Ratledge, 1972; Chen and Chang, 2013). Disruption of membrane structure impairs vital functions, such as the loss of ions, metabolites, lipids, and proteins, and the dissipation of the $\mathrm{pH}$ gradient and electrical potential. To overcome this toxicity issue, a clear understanding of the molecular mechanisms of interaction between yeast and alkanes could help to develop engineering strategies to improve microbial tolerance against alkane biofuel.

To tackle this toxicity issue caused by hydrocarbon biofuels, especially by alkanes, transcriptome analyses of S. cerevisiae have been accomplished, and suggested that $\mathrm{C} 9$ and $\mathrm{C} 10$ alkanes induced a wide range of rewiring of cellular metabolism, such as induction of efflux pumps, membrane modification, radical detoxification and energy supply (Ling et al., 2013). Since efflux pumps could possibly facilitate alkane secretion, and hence reduce the cytotoxicity, several studies have engineered the efflux pumps in S. cerevisiae. It is demonstrated that efflux pumps SNQ2P and PDR5P could reduce the intracellular levels of $\mathrm{C} 10$ and $\mathrm{C} 11$ alkanes and enhance the strain 
tolerance to alkanes. Upon $24 \mathrm{~h}$ exposure to $\mathrm{C} 10$ and $\mathrm{C} 11$, the amount of intracellular alkanes was lowered by 33 and 94.4\%, respectively (Ling et al., 2013). In addition, heterologous expression of $\mathrm{ABC} 2$ and $\mathrm{ABC} 3$ transporters from Y. lipolytica, which could utilize alkanes as the sole carbon source, significantly increased tolerance of $S$. cerevisiae against decane and undecane through maintaining lower intracellular alkane level. In particular, $\mathrm{ABC} 2$ transporter increased the tolerance limit of S. cerevisiae about 80 -fold against decane (Chen and Chang, 2013).

\section{The Lack of Metabolic Engineering Tools in Non-Model Oleaginous Yeast}

Besides S. cerevisiae, some oleaginous yeast strains have the ability to accumulate lipids to high levels, up to more than $20 \%$ of their biomass (Beopoulos et al., 2009; Munch et al., 2015). Among these oleaginous yeasts, Y. lipolytica is a unique host for biochemical production due to its abilities to accumulate high levels of lipids and utilize hydrophobic and waste carbon sources. To this end, $Y$. lipolytica has attracted great attention as a potential biofuel producing host. In the past decade, a series of genetic tools have been developed to transform plasmids, knock out genes, and develop both episomal and integrative expression cassettes to enable metabolic engineering approaches in Y. lipolytica. However, compared to the model yeast strain such as $S$. cerevisiae, several functions have not yet been achieved. For example, the tunable and high-level gene expression tools are still lacking in Y. lipolytica (Blazeck et al., 2014). Overall, the lack of molecular biology tools for tunable, modular, and high-throughput modification of genetic parts limits the use of $Y$. lipolytica for producing fatty acid-derived biofuels.

To this end, prior attempts have focused on designing strong constitutive promoters in Y. lipolytica by modifying the expression range of endogenous promoters through point mutations. For example, error-prone PCR of the native $S$. cerevisiae TEF promoter yielded a library of mutant promoters with a nearly 17-fold range in relative expression levels (Blazeck et al., 2013). Following this research, a hybrid promoter approach was developed to produce a novel library of highexpressing, tunable promoters in Y. lipolytica. The result revealed promoters in $Y$. lipolytica were enhancer limited, and such limitation can be partially or fully alleviated through the addition of tandem copies of upstream activation sequences (UASs) (Blazeck et al., 2011). Other genetic tools, such as CRISPR and one-step recombination of multiple genes (Gao et al., 2014), are also being developed for Y. lipolytica. All of these rational metabolic engineering efforts could significantly enhance the lipogenesis titers in $Y$. lipolytica. However, the resulting strain still suffered from decreased biomass generation rates. Recently, a rapid evolutionary metabolic engineering approach linked with a floating cell enrichment process was employed to improve lipogenesis rates, titers, and yields. Through this iterative process, researchers were able to ultimately improve yields from their prior strain by $55 \%$ to achieve production titers of $39.1 \mathrm{~g} / \mathrm{L}$ (Liu et al., 2015b). The highest lipid titer, $55 \mathrm{~g} / \mathrm{L}$, was achieved recently by simultaneous overexpression of delta- 9 stearoylCoA desaturase (SCD), acetyl-CoA carboxylase (ACC1), and diacylglyceride acyl-transferase (DGA1) in Y. lipolytica (Qiao et al., 2015).

\section{Other Potential Issues in Metabolic Engineering of Yeast to Produce Fatty Acid-Derived Biofuels}

\section{Difference of Fatty Acid Metabolism between $E$. coli and S. cerevisiae}

Beside $S$. cerevisiae and Y. lipolytica, the prokaryote model microorganism E. coli is another widely used industrial workhorse for producing fatty acid-derived biofuels. E. coli uses the type II FASs to catalyze FAB. The direct product of type II FASs is in the form of fatty acyl-ACPs, which need to be hydrolyzed by a thioesterase (TE) to release FFAs, and subsequently activated to fatty acyl-CoA by a fatty acyl-CoA ligase. Since this type of FASs was constructed by a series of mono-functional proteins that are discretely expressed from a series of separate genes, engineering on these structural genes was much easier than type I FASs. It is shown that when simply overexpressing the acyl-ACP thioesterase gene from $R$. communis in E. coli, the accumulation of FFAs could reach a high level of more than $2.0 \mathrm{~g} / \mathrm{L}$ at $48 \mathrm{~h}$, which was about 40-fold compared to the control strain (Zhang et al., 2011b). In comparison, by replacing the native promoters of $A C C 1$, FAS1, and FAS2 with strong and constitutive promoters in $S$. cerevisiae, their expression levels were increased about 7-16 fold, but the production of FFAs, fatty alcohols, and FAEEs were only increased by about 11-, 2-, and 4-fold, respectively, which is much lower than the improvement in E. coli (Lian and Zhao, 2014; Runguphan and Keasling, 2014). Therefore, the same strategy that works for E. coli may not work as well for yeast. Another example is the different behavior between the two species when engineering the $\beta$-oxidation pathway. A study in deletion of $P O X 1$, the first gene in the $\beta$-oxidation pathway in S. cerevisiae, did not improve fatty alcohol production titer (Runguphan and Keasling, 2014). But in combination with the deletion of other genes in the fatty acid degradation pathway, FFAs have been produced at high titers $(8.6 \mathrm{~g} / \mathrm{L}$ ) in E. coli (Xu et al., 2013). Such discrepancy could probably result from the more complex regulatory mechanisms and cellular compartmentalization in S. cerevisiae.

\section{Balancing Metabolic Pathways in Yeast}

The strategies for balancing the enzyme activities and expression to tune metabolic flux are important to improve the productivity. In E. coli, a dynamic sensor-regulator system (DSRS) to produce fatty acid-based products has been established, and demonstrated its use for biodiesel production (Zhang et al., 2012). By incorporating the Bacillus subtilis trans-regulatory protein fapR and the cis-regulatory element fapO, a malonyl-CoA responsive sensor has been developed which holds great promise in overcoming critical pathway limitations and optimizing titers and yields of target products ( $\mathrm{Xu}$ et al., 2014). These sensors 
reported in E. coli could potentially control and optimize carbon flux, which leads to robust biosynthetic pathways. However, the strategy of balancing metabolic pathways has not yet been fully explored in yeast strains such as $S$. cerevisiae. The challenge in building a regulatory system in yeast is the lack of engineered promoters and functionally expression genetic parts under the tightly regulated genetic background, since the DSRS requires the combination of various kinds of promoters that can both detect key intermediates in the synthesis cascade and control gene expression to improve production of the desired chemicals. To establish a similar DSRS in yeast, the frontier of eukaryotic promoters needs to be further explored to develop new systems such as RNA based response and regulatory elements.

\section{Optimizing Fermentation Conditions to Further Improve the Productivity}

Process optimization, aiming at maintaining optimal and homogenous reaction conditions, minimizing microbial stress exposure, and enhancing metabolic activities, plays vital roles in achieving high productivity of fatty acid-derived biofuels. By identifying the most relevant process parameters that affect product yield and quality, the fermentation strategy could be optimized and maximize the productivity of a metabolically engineered strain. Recently, the importance of optimizing fermentation conditions has been demonstrated. In a research project related to the enhancement of FAEE production in $S$. cerevisiae, nitrogen limiting culture was used to achieve an immediate increase (over 3.5-fold) in the titer of FAEEs compared to the control culture conditions (Thompson and Trinh, 2014). In addition, switching from the batch mode to fed-batch, resting cell fermentation mode, the highest titer of 1hexadecanol produced by an engineered $S$. cerevisiae strain could reach $1111 \mathrm{mg} / \mathrm{L}$, a 10 -fold increase compared to the previous report on engineering yeast for fatty alcohol production (Hong and Nielsen, 2012). However, the systemic characterization and improvement of fermentation conditions are still required for improving the biofuel productivity, especially for pilot scale production.

\section{References}

Ambroziak,J., and Henry, S. A. (1994). INO2 and INO4 gene products, positive regulators of phospholipid biosynthesis in Saccharomyces cerevisiae, form a complex that binds to the INOl promoter. J. Biol. Chem. 269, 6.

Antoni, D., Zverlov, V. V., and Schwarz, W. H. (2007). Biofuels from microbes. Appl. Microbiol. Biotechnol. 77, 23-35. doi: 10.1007/s00253-0071163-x

BCC Research LLC. (2013). Global Markets for Oleochemical Fatty Acids. Wellesley, MA. Available online at: http://www.bccresearch.com/marketresearch/chemicals/oleochemical-fatty-acids-markets-chm062a.html

Beld, J., Lee, D. J., and Burkart, M. D. (2015). Fatty acid biosynthesis revisited: structure elucidation and metabolic engineering. Mol. Biosyst. 11, 38-59. doi: 10.1039/C4MB00443D

Beopoulos, A., Cescut, J., Haddouche, R., Uribelarrea, J. L., Molina-Jouve, C., and Nicaud, J. M. (2009). Yarrowia lipolytica as a model for biooil production. Prog. Lipid Res. 48, 375-387. doi: 10.1016/j.plipres.2009. 08.005

\section{Concluding Remarks}

To engineer yeast for production of a wide range of fatty acid-derived biofuels, bottlenecks such as the limited supply of FAB precursor and cofactors, the tight regulations of fatty acid metabolism, and the product toxicity to host cells needs to be overcome to achieve high productivity. In order to tackle these limitations, extensive efforts on metabolic engineering have been made to improve the productivity of fatty acid-derived biofuels in yeast strains. Until now, most of the researches focused on the improvement of precursors and co-factor supply in FAB. After engineering both the heterologous and endogenous pathways, significant increase of the productivity of fatty acids-derived chemicals has been achieved. There is also an interesting trend of engineering the regulatory elements of yeast metabolism to relieve the tight regulation on some rate limiting enzymes and improve the productivity. In addition to all of these successful strategies, there are other possible approaches that could also help to improve the yeast-based production of fatty acid-derived biofuels, such as the design of dynamic in vivo sensor system. To date, no single synthetic biology approach could completely meet the demands to develop an almighty host for producing fatty acid-derived biofuels. Therefore, the integration of "omics" analysis [e.g., genomics (Cherry et al., 2012; Lee et al., 2013), transcriptomics (Wang et al., 2009; Rossouw et al., 2010), proteomics (Chen and Snyder, 2010; Rossouw et al., 2010), metabolomics (Smedsgaard and Nielsen, 2005; Jewison et al., 2012), and fluxomics (Feng and Zhao, 2013; Winter and Kromer, 2013)] with the rational applications of appropriate biomolecular engineering toolbox could make steady progress toward the goal of engineering yeast to be a more suitable host for producing fatty acid-derived biofuels.

\section{Acknowledgments}

We thank the writing center in Virginia Tech for improving the language of the paper. This study was supported by start-up fund (\#175323) from Virginia Tech.

Bernard, A., Domergue, F., Pascal, S., Jetter, R., Renne, C., Faure, J. D., et al. (2012). Reconstitution of plant alkane biosynthesis in yeast demonstrates that Arabidopsis ECERIFERUM1 and ECERIFERUM3 are core components of a very-long-chain alkane synthesis complex. Plant Cell 24, 3106-3118. doi: 10.1105/tpc.112.099796

Blazeck, J., Hill, A., Liu, L., Knight, R., Miller, J., Pan, A., et al. (2014). Harnessing Yarrowia lipolytica lipogenesis to create a platform for lipid and biofuel production. Nat. Commun. 5, 3131. doi: 10.1038/ncomms4131

Blazeck, J., Liu, L., Redden, H., and Alper, H. (2011). Tuning gene expression in Yarrowia lipolytica by a hybrid promoter approach. Appl. Environ. Microbiol. 77, 7905-7914. doi: 10.1128/AEM.05763-11

Blazeck, J., Reed, B., Garg, R., Gerstner, R., Pan, A., Agarwala, V., et al. (2013). Generalizing a hybrid synthetic promoter approach in Yarrowia lipolytica. Appl. Microbiol. Biotechnol. 97, 3037-3052. doi: 10.1007/s00253-0124421-5

BP p.l.c. (2014). BP Statistical Review of World Energy, 2014. London. Availabel online at: http://www.bp.com/en/global/corporate/about-bp/energyeconomics/statistical-review-of-world-energy.html 
Bruinenberg, P. M., Van Dijken, J. P., and Scheffers, W. A. (1983). A theoretical analysis of NADPH production and consumption in yeast. J. Gen. Microbiol. 129, 12. doi: 10.1099/00221287-129-4-953

Buijs, N. A., Zhou, Y. J., Siewers, V., and Nielsen, J. (2014). Long-chain alkane production by the yeast Saccharomyces cerevisiae. Biotechnol. Bioeng. 112, 1275-1279. doi: 10.1002/bit.25522

Cao, Y. X., Xiao, W. H., Liu, D., Zhang, J. L., Ding, M. Z., and Yuan, Y. J. (2015). Biosynthesis of odd-chain fatty alcohols in Escherichia coli. Metab. Eng. 29, 119-123. doi: 10.1016/j.ymben.2015.03.005

Cardenas, J., and Da Silva, N. A. (2014). Metabolic engineering of Saccharomyces cerevisiae for the production of triacetic acid lactone. Metab. Eng. 25, 194-203. doi: 10.1016/j.ymben.2014.07.008

Chan, D. I., and Vogel, H. J. (2010). Current understanding of fatty acid biosynthesis and the acyl carrier protein. Biochem. J. 430, 1-19. doi: 10.1042/BJ20100462

Chen, B. L. H., and Chang, M. W. (2013). Transporter engineering for improved tolerance against alkane biofuels in Saccharomyces cerevisiae. Biotechnol. Biofuels 6, 10. doi: 10.1186/1754-6834-6-21

Chen, L., Zhang, J., Lee, J., and Chen, W. N. (2014). Enhancement of free fatty acid production in Saccharomyces cerevisiae by control of fatty acyl-CoA metabolism. Appl. Microbiol. Biotechnol. 98, 6739-6750. doi: 10.1007/s00253014-5758-8

Chen, R., and Snyder, M. (2010). Yeast proteomics and protein microarrays. J. Proteomics 73, 2147-2157. doi: 10.1016/j.jprot.2010.08.003

Chen, W. N., and Tan, K. Y. (2013). "Malonate uptake and metabolism in Saccharomyces cerevisiae." Appl. Biochem. Biotechnol. 171, 44-62. doi: 10.1007/s12010-013-0334-8

Chen, Y., Bao, J., Kim, I. K., Siewers, V., and Nielsen, J. (2014a). Coupled incremental precursor and co-factor supply improves 3-hydroxypropionic acid production in Saccharomyces cerevisiae. Metab. Eng. 22, 104-109. doi: 10.1016/j.ymben.2014.01.005

Chen, Y., Daviet, L., Schalk, M., Siewers, V., and Nielsen, J. (2013). Establishing a platform cell factory through engineering of yeast acetyl-CoA metabolism. Metab. Eng. 15, 48-54. doi: 10.1016/j.ymben.2012.11.002

Chen, Y., Siewers, V., and Nielsen, J. (2012). Profiling of cytosolic and peroxisomal acetyl-CoA metabolism in Saccharomyces cerevisiae. PLoS ONE 7:e42475. doi: 10.1371/journal.pone.0042475

Cherry, J. M., Hong, E. L., Amundsen, C., Balakrishnan, R., Binkley, G., Chan, E. T., et al. (2012). Saccharomyces genome database: the genomics resource of budding yeast. Nucleic Acids Res. 40, D700-D705. doi: 10.1093/nar/gkr1029

Choi, Y. J., and Lee, S. Y. (2013). Microbial production of short-chain alkanes. Nature 502, 571-574. doi: 10.1038/nature12536

Chu, H. S., Kim, Y. S., Lee, C. M., Lee, J. H., Jung, W. S., Ahn, J. H., et al. (2015). Metabolic engineering of 3-hydroxypropionic acid biosynthesis in Escherichia coli. Biotechnol. Bioeng. 112, 356-364. doi: 10.1002/bit.25444

Clomburg, J. M., Blankschien, M. D., Vick, J. E., Chou, A., Kim, S., and Gonzalez, R. (2015). Integrated engineering of beta-oxidation reversal and omega-oxidation pathways for the synthesis of medium chain omega-functionalized carboxylic acids. Metab. Eng. 28, 202-212. doi: 10.1016/j.ymben.2015.01.007

Connor, M. R., and Liao, J. C. (2009). Microbial production of advanced transportation fuels in non-natural hosts. Curr. Opin. Biotechnol. 20, 307-315. doi: 10.1016/j.copbio.2009.04.002

Crabtree, H. G. (1928). The carbohydrate metabolism of certain pathological overgrowths. Biochem. J. 22, 10.

Cronan, J. E. Jr., and Waldrop, G. L. (2002). Multi-subunit acetyl-CoA carboxylases. Prog. Lipid. Res. 41, 407-435. doi: 10.1016/s0163-7827(02) 00007-3

de Jong, B. W., Shi, S., Siewers, V., and Nielsen, J. (2014). Improved production of fatty acid ethyl esters in Saccharomyces cerevisiae through up-regulation of the ethanol degradation pathway and expression of the heterologous phosphoketolase pathway. Microb. Cell Fact. 13:39. doi: 10.1186/14752859-13-39

Egner, R., Thumm, M., Straub, M., Simeon, A., Schüller, H. J., and Wolf, D. H. (1993). Tracing intracellular proteolytic pathways. Proteolysis of fatty acid synthase and other cytoplasmic proteins in the yeast Saccharomyces cerevisiae. J. Biol. Chem. 268, 8.

Elbahloul, Y., and Steinbuchel, A. (2010). Pilot-scale production of fatty acid ethyl esters by an engineered Escherichia coli strain harboring the p(Microdiesel) plasmid. Appl. Environ. Microbiol. 76, 4560-4565. doi: 10.1128/AEM. 00515-10

Eriksen, D. T., HamediRad, M., Yuan, Y., and Zhao, H. (2015). Orthogonal fatty acid biosynthetic pathway improves fatty acid ethyl ester production in Saccharomyces cerevisiae. ACS Synth. Biol. doi: 10.1021/sb500319p. [Epub ahead of print].

Feng, X., Lian, J., and Zhao, H. (2014). Metabolic engineering of Saccharomyces cerevisiae to improve 1-hexadecanol production. Metab. Eng. 27C, 10-19. doi: 10.1016/j.ymben.2014.10.001

Feng, X., and Zhao, H. (2013). Investigating xylose metabolism in recombinant Saccharomyces cerevisiae via 13C metabolic flux analysis. Microb. Cell Fact. 12:114. doi: 10.1186/1475-2859-12-114

Floudas, C. A., Elia, J. A., and Baliban, R. C. (2012). Hybrid and single feedstock energy processes for liquid transportation fuels: a critical review. Comput. Chem. Eng. 41, 24-51. doi: 10.1016/j.compchemeng.2012.02.008

Fukuda, H., Kond, A., and Noda, H. (2001). Biodiesel fuel production by transesterification of oils. J. Biosci. Bioeng. 92, 12. doi: 10.1016/S13891723(01)80288-7

Gao, S., Han, L., Zhu, L., Ge, M., Yang, S., Jiang, Y., et al. (2014). One-step integration of multiple genes into the oleaginous yeast Yarrowia lipolytica. Biotechnol. Lett. 36, 2523-2528. doi: 10.1007/s10529-014-1634-y

Gill, C. O., and Ratledge, C. (1972). Toxicity of n-Alkanes, n-Alk-1-enes, n-Alkan1-ols and n-Alkyl-1-bromides towards Yeasts. J. Gen. Microbiol. 72, 8. doi: 10.1099/00221287-72-1-165

Guo, Z. P., Zhang, L., Ding, Z. Y., and Shi, G. Y. (2011). Minimization of glycerol synthesis in industrial ethanol yeast without influencing its fermentation performance. Metab. Eng. 13, 49-59. doi: 10.1016/j.ymben.2010. 11.003

Hamelinck, C. N., and Faaij, A. P. C. (2006). Outlook for advanced biofuels. Energy Policy 34, 3268-3283. doi: 10.1016/j.enpol.2005.06.012

Henke, B., Girzalsky, W., Berteaux-Lecellier, V., and Erdmann, R. (1998). IDP3 encodes a peroxisomal NADP-dependent isocitrate dehydrogenase required for the beta-oxidation of unsaturated fatty acids. J. Biol. Chem. 273, 10. doi: 10.1074/jbc.273.6.3702

Hiltunen, J. K., Schonauer, M. S., Autio, K. J., Mittelmeier, T. M., Kastaniotis, A. J., and Dieckmann, C. L. (2009). Mitochondrial fatty acid synthesis type II: more than just fatty acids. J. Biol. Chem. 284, 9011-9015. doi: 10.1074/jbc.R800068200

Hong, K. K., and Nielsen, J. (2012). Metabolic engineering of Saccharomyces cerevisiae: a key cell factory platform for future biorefineries. Cell. Mol. Life Sci. 69, 2671-2690. doi: 10.1007/s00018-012-0945-1

Inui, H., Ono, K., Miyatake, K., Nakano, Y., and Kitaoka, S. (1987). Purification and characterization of pyruvate:NADP+ oxidoreductase in Euglena gracilis. J. Biol. Chem. 262, 6.

Jewison, T., Knox, C., Neveu, V., Djoumbou, Y., Guo, A. C., Lee, J., et al. (2012). YMDB: the Yeast Metabolome Database. Nucleic Acids Res. 40, D815-D820. doi: 10.1093/nar/gkr916

Jia, C., Li, M., Li, J., Zhang, J., Zhang, H., Cao, P., et al. (2015). Structural insights into the catalytic mechanism of aldehyde-deformylating oxygenases. Protein Cell 6, 55-67. doi: 10.1007/s13238-014-0108-2

Jing, F., Cantu, D. C., Tvaruzkova, J., Chipman, J. P., Nikolau, B. J., YandeauNelson, M. D., et al. (2011). Phylogenetic and experimental characterization of an acyl-ACP thioesterase family reveals significant diversity in enzymatic specificity and activity. BMC Biochem. 12:44. doi: 10.1186/1471-209112-44

Jones, J. A., Toparlak, O. D., and Koffas, M. A. (2014). Metabolic pathway balancing and its role in the production of biofuels and chemicals. Curr. Opin. Biotechnol. 33, 52-59. doi: 10.1016/j.copbio.2014.11.013

Kissin, Y. V. (1987). Catagenesis and composition of petroleum: origin of nalkanes and isoalkanes in petroleum crudes. Geochim. Cosmochim. Acta 51, 13. doi: 10.1016/0016-7037(87)90296-1

Kozak, B. U., van Rossum, H. M., Benjamin, K. R., Wu, L., Daran, J. M., Pronk, J. T., et al. (2014). Replacement of the Saccharomyces cerevisiae acetyl-CoA synthetases by alternative pathways for cytosolic acetyl-CoA synthesis. Metab. Eng. 21, 46-59. doi: 10.1016/j.ymben.2013.11.005

Krivoruchko, A., Zhang, Y., Siewers, V., Chen, Y., and Nielsen, J. (2015). Microbial acetyl-CoA metabolism and metabolic engineering. Metab. Eng. 28, 28-42. doi: 10.1016/j.ymben.2014.11.009 
Lee, S., Lim, W. A., and Thorn, K. S. (2013). Improved blue, green, and red fluorescent protein tagging vectors for S. cerevisiae. PLoS ONE 8:e67902. doi: 10.1371/journal.pone.0067902

Lee, S. Y., Kim, H. M., and Cheon, S. (2014). Metabolic engineering for the production of hydrocarbon fuels. Curr. Opin. Biotechnol. 33C, 15-22. doi: 10.1016/j.copbio.2014.09.008

Lennen, R. M., and Pfleger, B. F. (2013). Microbial production of fatty acidderived fuels and chemicals. Curr. Opin. Biotechnol. 24, 1044-1053. doi: 10.1016/j.copbio.2013.02.028

Li, X., Guo, D., Cheng, Y., Zhu, F., Deng, Z., and Liu, T. (2014). Overproduction of fatty acids in engineered Saccharomyces cerevisiae. Biotechnol. Bioeng. 111, 1841-1852. doi: 10.1002/bit.25239

Lian, J., Si, T., Nair, N. U., and Zhao, H. (2014). Design and construction of acetylCoA overproducing Saccharomyces cerevisiae strains. Metab. Eng. 24, 139-149. doi: 10.1016/j.ymben.2014.05.010

Lian, J., and Zhao, H. (2014). Recent advances in biosynthesis of fatty acids derived products in Saccharomyces cerevisiae via enhanced supply of precursor metabolites. J. Ind. Microbiol. Biotechnol. 42, 437-451. doi: 10.1007/s10295014-1518-0

Lian, J., and Zhao, H. (2015). Reversal of the beta-oxidation cycle in Saccharomyces cerevisiae for production of fuels and chemicals. ACS Synth. Biol. 4, 332-341. doi: $10.1021 / \mathrm{sb} 500243 \mathrm{c}$

Ling, H., Chen, B., Kang, A., Lee, J. M., and Chang, M. W. (2013). Transcriptome response to alkane biofuels in Saccharomyces cerevisiae identification of efflux pumps involved in alkane tolerance. Biotechnol. Biofuels 6, 10. doi: 10.1186/1754-6834-6-95

Liu, L., Pan, A., Spofford, C., Zhou, N., and Alper, H. S. (2015b). An evolutionary metabolic engineering approach for enhancing lipogenesis in Yarrowia lipolytica. Metab. Eng. 29, 36-45. doi: 10.1016/j.ymben.2015.02.003

Liu, Q., Wu, K., Cheng, Y., Lu, L., Xiao, E., Zhang, Y., et al. (2015a). Engineering an iterative polyketide pathway in Escherichia coli results in single-form alkene and alkane overproduction. Metab. Eng. 28, 82-90. doi: 10.1016/j.ymben.2014.12.004

Liu, R., Zhu, F., Lu, L., Fu, A., Lu, J., Deng, Z., et al. (2014). Metabolic engineering of fatty acyl-ACP reductase-dependent pathway to improve fatty alcohol production in Escherichia coli. Metab. Eng. 22, 10-21. doi: 10.1016/j.ymben.2013.12.004

Minard, K. I., and McAlister-Henn, L. (2005). Sources of NADPH in yeast vary with carbon source. J. Biol. Chem. 280, 39890-39896. doi: 10.1074/jbc.M509461200

Munch, G., Sestric, R., Sparling, R., Levin, D. B., and Cicek, N. (2015). Lipid production in the under-characterized oleaginous yeasts, Rhodosporidium babjevae and Rhodosporidium diobovatum, from biodiesel-derived waste glycerol. Bioresour. Technol. 185C, 49-55. doi: 10.1016/j.biortech.2015. 02.051

Pasztor, A., Kallio, P., Malatinszky, D., Akhtar, M. K., and Jones, P. R. (2015). A synthetic $\mathrm{O} 2$-tolerant butanol pathway exploiting native fatty acid biosynthesis in Escherichia coli. Biotechnol. Bioeng. 112, 120-128. doi: 10.1002/bit.25324

Peralta-Yahya, P. P., Ouellet, M., Chan, R., Mukhopadhyay, A., Keasling, J. D., and Lee, T. S. (2011). Identification and microbial production of a terpene-based advanced biofuel. Nat. Commun. 2, 483. doi: 10.1038/ncomms1494

Peralta-Yahya, P. P., Zhang, F., del Cardayre, S. B., and Keasling, J. D. (2012). Microbial engineering for the production of advanced biofuels. Nature 488, 320-328. doi: $10.1038 /$ nature11478

Pfeiffer, T., and Morley, A. (2014). An evolutionary perspective on the Crabtree effect. Front. Mol. Biosci. 1:17. doi: 10.3389/fmolb.2014.00017

Pfleger, B. F., Gossing, M., and Nielsen, J. (2015). Metabolic engineering strategies for microbial synthesis of oleochemicals. Metab. Eng. 29, 1-11. doi: 10.1016/j.ymben.2015.01.009

Postma, E., Verduyn, C., Scheffers, W. A., and Van Dijken, J. P. (1989). Enzymic analysis of the crabtree effect in glucose-limited chemostat cultures of Saccharomyces cerevisiae. 55, 10.

Qiao, K., Imam Abidi, S. H., Liu, H., Zhang, H., Chakraborty, S., Watson, N., et al. (2015). Engineering lipid overproduction in the oleaginous yeast Yarrowia lipolytica. Metab. Eng. 29, 56-65. doi: 10.1016/j.ymben.2015.02.005

Rossouw, D., van den Dool, A. H., Jacobson, D., and Bauer, F. F. (2010). Comparative transcriptomic and proteomic profiling of industrial wine yeast strains. Appl. Environ. Microbiol. 76, 3911-3923. doi: 10.1128/AEM.00586-10
Runguphan, W., and Keasling, J. D. (2014). Metabolic engineering of Saccharomyces cerevisiae for production of fatty acid-derived biofuels and chemicals. Metab. Eng. 21, 103-113. doi: 10.1016/j.ymben.2013.07.003

Schirmer, A., Rude, M. A., Li, X., Popova, E., and del Cardayre, S. B. (2010). Microbial biosynthesis of alkanes. Science 329, 5. doi: 10.1126/science.11 87936

Schüller, H. J., Schorr, R., Hoffmann, B., and Schweizer, E. (1992). Regulatory gene INO4 of yeast phospholipid biosynthesis is positively autoregulated and functions as a transactivator of fatty acid synthase genes FAS1 and FAS2 from Saccharomyces cerevisiae. Nucleic Acids Res. 20, 7. doi: 10.1093/nar/20.22.5955

Schüller, H. J., Schütz, A., Knab, S., Hoffmann, B., and Schweizer, E. (1994). Importance of general regulatory factors Raplp, Abflp and Reblp for the activation of yeast fatty acid synthase genes FAS1 and FAS2. Eur. J. Biochem. 225, 10. doi: 10.1111/j.1432-1033.1994.00213.x

Shi, L., and Tu, B. P. (2015). Acetyl-CoA and the regulation of metabolism: mechanisms and consequences. Curr. Opin. Cell Biol. 33C, 125-131. doi: 10.1016/j.ceb.2015.02.003

Shi, S., Chen, Y., Siewers, V., and Nielsen, J. (2014b). Improving production of malonyl coenzyme A-derived metabolites by abolishing Snf1-dependent regulation of Acc1. MBio 5, e01130-e01114. doi: 10.1128/mBio.01130-14

Shi, S., Valle-Rodriguez, J. O., Siewers, V., and Nielsen, J. (2014a). Engineering of chromosomal wax ester synthase integrated Saccharomyces cerevisiae mutants for improved biosynthesis of fatty acid ethyl esters. Biotechnol. Bioeng. 111, 1740-1747. doi: 10.1002/bit.25234

Smedsgaard, J., and Nielsen, J. (2005). Metabolite profiling of fungi and yeast: from phenotype to metabolome by MS and informatics. J. Exp. Bot. 56, 273-286. doi: $10.1093 / \mathrm{jxb} / \mathrm{eri} 068$

Sonderegger, M., Schumperli, M., and Sauer, U. (2004). Metabolic engineering of a phosphoketolase pathway for pentose catabolism in Saccharomyces cerevisiae. Appl. Environ. Microbiol. 70, 2892-2897. doi: 10.1128/AEM.70.5.28922897.2004

Steen, E. J., Kang, Y., Bokinsky, G., Hu, Z., Schirmer, A., McClure, A., et al. (2010). Microbial production of fatty-acid-derived fuels and chemicals from plant biomass. Nature 463, 559-562. doi: 10.1038/nature08721

Strijbis, K., and Distel, B. (2010). Intracellular acetyl unit transport in fungal carbon metabolism. Eukaryotic Cell 9, 1809-1815. doi: 10.1128/EC.00172-10

Tai, M., and Stephanopoulos, G. (2013). Engineering the push and pull of lipid biosynthesis in oleaginous yeast Yarrowia lipolytica for biofuel production. Metab. Eng. 15, 1-9. doi: 10.1016/j.ymben.2012.08.007

Tai, Y. S., Xiong, M., and Zhang, K. (2015). Engineered biosynthesis of medium-chain esters in Escherichia coli. Metab. Eng. 27, 20-28. doi: 10.1016/j.ymben.2014.10.004

Takamura, Y., and Nomura, G. (1988). Changes in the intracellular concentration of acetyl-CoA and malonyl-CoA in relation to the carbon and energy metabolism of Escherichia coli K12. J. Gen. Microbiol. 134, 5. doi: 10.1099/00221287-134-8-2249

Tang, X., Feng, H., and Chen, W. N. (2013). Metabolic engineering for enhanced fatty acids synthesis in Saccharomyces cerevisiae. Metab. Eng. 16, 95-102. doi: 10.1016/j.ymben.2013.01.003

Tee, T. W., Chowdhury, A., Maranas, C. D., and Shanks, J. V. (2014). Systems metabolic engineering design: fatty acid production as an emerging case study. Biotechnol. Bioeng. 111, 849-857. doi: 10.1002/bit.25205

Tehlivets, O., Scheuringer, K., and Kohlwein, S. D. (2007). Fatty acid synthesis and elongation in yeast. Biochim. Biophys. Acta 1771, 255-270. doi: 10.1016/j.bbalip.2006.07.004

Thompson, R. A., and Trinh, C. T. (2014). Enhancing fatty acid ethyl ester production in Saccharomyces cerevisiae through metabolic engineering and medium optimization. Biotechnol. Bioeng. 111, 2200-2208. doi: 10.1002/bit. 25292

Trotter, P. J. (2001). The genetics of fatty acid metabolism in Sacchromyses cerevisiae. Аnnu. Rev. Nutr. 21, 23. doi: 10.1146/annurev.nutr.21.1.97

Valle-Rodríguez, J. O., Shi, S., Siewers, V., and Nielsen, J. (2014). Metabolic engineering of Saccharomyces cerevisiae for production of fatty acid ethyl esters, an advanced biofuel, by eliminating non-essential fatty acid utilization pathways. Appl. Energy 115, 226-232. doi: 10.1016/j.apenergy.2013.10.003

van Roermund, C. W., Hettema, E. H., Kal, A. J., van den Berg, M., Tabak, H. F., and Wanders, R. J. (1998). Peroxisomal beta-oxidation of polyunsaturated fatty acids in Saccharomyces cerevisiae: isocitrate dehydrogenase provides 
NADPH for reduction of double bonds at even positions. EMBO J. 17, 11. doi: 10.1093/emboj/17.3.677

Verho, R., Londesborough, J., Penttila, M., and Richard, P. (2003). Engineering redox cofactor regeneration for improved pentose fermentation in Saccharomyces cerevisiae. Appl. Environ. Microbiol. 69, 5892-5897. doi: 10.1128/AEM.69.10.5892-5897.2003

Wang, Y., Chen, H., and Yu, O. (2014). A plant malonyl-CoA synthetase enhances lipid content and polyketide yield in yeast cells. Appl. Microbiol. Biotechnol. 98, 5435-5447. doi: 10.1007/s00253-014-5612-Z

Wang, Z., Gerstein, M., and Snyder, M. (2009). RNA-Seq: a revolutionary tool for transcriptomics. Nat. Rev. Genet. 10, 7. doi: 10.1038/nrg2484

Wattanachaisaereekul, S., Lantz, A. E., Nielsen, M. L., and Nielsen, J. (2008). Production of the polyketide 6-MSA in yeast engineered for increased malonylCoA supply. Metab. Eng. 10, 246-254. doi: 10.1016/j.ymben.2008.04.005

Winter, G., and Kromer, J. O. (2013). Fluxomics - connecting 'omics analysis and phenotypes. Environ. Microbiol. 15, 1901-1916. doi: 10.1111/1462-2920.12064

Xu, P., Gu, Q., Wang, W., Wong, L., Bower, A. G., Collins, C. H., et al. (2013). Modular optimization of multi-gene pathways for fatty acids production in $E$. coli. Nat. Commun. 4, 1409. doi: 10.1038/ncomms 2425

Xu, P., Wang, W., Li, L., Bhan, N., Zhang, F., and Koffas, M. A. (2014). Design and kinetic analysis of a hybrid promoter-regulator system for malonyl-CoA sensing in Escherichia coli. ACS Chem. Biol. 9, 451-458. doi: $10.1021 / \mathrm{cb} 400623 \mathrm{~m}$

Yan, Y., and Liao, J. C. (2009). Engineering metabolic systems for production of advanced fuels. J. Ind. Microbiol. Biotechnol. 36, 471-479. doi: 10.1007/s10295009-0532-0

Youngquist, J. T., Lennen, R. M., Ranatunga, D. R., Bothfeld, W. H., Marner, W. D. 2nd., and Pfleger, B. F. (2012). Kinetic modeling of free fatty acid production in Escherichia coli based on continuous cultivation of a plasmid free strain. Biotechnol. Bioeng. 109, 1518-1527. doi: 10.1002/bit.24420

Yu, K. O., Jung, J., Kim, S. W., Park, C. H., and Han, S. O. (2012). Synthesis of FAEEs from glycerol in engineered Saccharomyces cerevisiae using endogenously produced ethanol by heterologous expression of an unspecific bacterial acyltransferase. Biotechnol. Bioeng. 109, 110-115. doi: 10.1002/bit.23311

Zaidi, N., Swinnen, J. V., and Smans, K. (2012). ATP-citrate lyase: a key player in cancer metabolism. Cancer Res. 72, 3709-3714. doi: 10.1158/0008-5472.CAN$11-4112$

Zhang, F., Carothers, J. M., and Keasling, J. D. (2012). Design of a dynamic sensorregulator system for production of chemicals and fuels derived from fatty acids. Nat. Biotechnol. 30, 354-359. doi: 10.1038/nbt.2149

Zhang, F., Rodriguez, S., and Keasling, J. D. (2011a). Metabolic engineering of microbial pathways for advanced biofuels production. Curr. Opin. Biotechnol. 22, 775-783. doi: 10.1016/j.copbio.2011.04.024

Zhang, H., Liu, Q., Cao, Y., Feng, X., Zheng, Y., Zou, H. et al. (2014). Microbial production of sabinene-a new terpene-based precursor of advanced biofuel. Microb. Cell Fact. 13:20. doi: 10.1186/1475-2859-13-20

Zhang, X., Li, M., Agrawal, A., and San, K. Y. (2011b). Efficient free fatty acid production in Escherichia coli using plant acyl-ACP thioesterases. Metab. Eng. 13, 713-722. doi: 10.1016/j.ymben.2011.09.007

Zhou, Y. J., Buijs, N. A., Siewers, V., and Nielsen, J. (2014). Fatty acid-derived biofuels and chemicals production in Saccharomyces cerevisiae. Front. Bioeng. Biotechnol. 2:32. doi: 10.3389/fbioe.2014.00032

Conflict of Interest Statement: The authors declare that the research was conducted in the absence of any commercial or financial relationships that could be construed as a potential conflict of interest.

Copyright (c) 2015 Sheng and Feng. This is an open-access article distributed under the terms of the Creative Commons Attribution License (CC BY). The use, distribution or reproduction in other forums is permitted, provided the original author(s) or licensor are credited and that the original publication in this journal is cited, in accordance with accepted academic practice. No use, distribution or reproduction is permitted which does not comply with these terms. 